\title{
DEVELOPED CRITERIA TO IMPROVE PILOT REPORTING OF AIRPLANE VORTEX ENCOUNTERS
}

\author{
Aziz AL-MAHADIN (iD) ${ }^{1,}$, Serdar DALKILIC (1D ${ }^{2}$ \\ ${ }^{1}$ Department of Aviation Maintenance/Faculty of Engineering Technology and Science, \\ Higher Colleges of Technology, Dubai, UAE \\ ${ }^{2}$ Department of Aviation Maintenance/Faculty of Engineering Technology and Science, \\ Higher Colleges of TechnologyuDhabi, UAE
}

Received 24 March 2019; accepted 27 January 2020

\begin{abstract}
Leading airplane vortices can be hazardous to following airplanes. The regulated minimum separations between following and leading airplanes are sometimes overjudged, hence causing reduction in the capacity of airports. In other instances, they are underjudged and subsequently causing airplane incidences. A vital contribution to the establishment and adjustment of vortex-related minimum airplane separations rely on the identification of vortex encounters through pilot reporting with a manual analysis of flight data from FDRs (flight data recorders). This current process relies on judgment of both the pilot and the airline analysist. Hence, it is subjective and sometimes lacks the required accuracy. Therefore, it is desirable to set a number of criteria, which can be utilized to evaluate the accuracy of wake vortex encounter identification. These criteria can save time, and are both accurate and simple. This study investigates 54 pilot reports of flight events to establish a set of criteria that enable concerned aviation organizations to confirm airplane vortex encounters with higher accuracy. This also helps airlines and aviation stakeholders to introduce new regulations and enhancements such as pilots and FDR analysts training on vortex identification. Such measures will enhance safety, improve aviation operation efficiency and allow revision of vortex-separation regulations.
\end{abstract}

Keywords: pilot reporting, flight events, identification of wake vortex encounter, flight data, flight data recorders (FDRs), wake vortex separation distance.

\section{Introduction}

Airplane wing vortices can be hazardous to a following airplane. This can be very dangerous at low altitude and during landing/take-off where the airplane separations are low. The impacts of airplane vortices and the associated separations have increased with the growth of air traffic and the use of larger airplanes. The two biggest commercial aircraft manufacturers Boeing and Airbus market research between 2012 and 2031 predicts a continuing growth in the aviation market. For example, 20-year world annual traffic growth of $4.7 \%$ and $5.0 \%$ are forecasted by Airbus and Boeing respectively. The number of passengers travelled by air was above 3 billion in 2011 and is forecasted to be above 7.5 billion in 2031 (Global market forecast 2012-2031, 2012; Current, 2012). This growth demands enhancing air safety, improving air travel effectiveness and increasing capacity of airports. This situation requires specialists and investigators to find better ways to analyse flight data and to identify the various flight events including wake vortex encounters.

To reduce the danger of a leading airplane vortex, following airplanes are required to wait during departure/ arrival until wing vortices generated by a previous airplane have diminished or pushed away from the flight path (Vortex avoidance procedures, 2017). The existing imposed standard separations are established mainly on airplane weight classes (Safety and flight operations: loss of control in-flight accident analysis, 2015; Hinton, 1997). Critchley and Foot (1991) have investigated these standard separations by analysing data from various airplane vortex encounters. They concluded that in many cases standard separations are not appropriate. The imposed separations, in some cases, are conservative causing a significant reduction in the capacity of airports. Conversely, some dangerous airplane vortices are underestimated and

*Corresponding author. E-mail: aalmahadin@hct.ac.ae 
can cause harm to following airplanes. Serious accidents caused by airplane vortices have been reported (Safety and flight operations: loss of control in-flight accident analysis, 2015; Critchley \& Foot, 1991). They could have been prevented if regulations had been established with better safety standards and with improved separations.

Several theoretical studies in the open literature can be found which investigated the generation of wake vortex, the behaviour of aircraft that encounter wake vortices and the adverse effects of wake vortex on flight safety (Höhne, Fuhrmann, \& Luckner, 2004; Chernyshev, Gaifullin, \& Sviridenko, 2014; Huang, Zhang, \& Cui, 2017; Aircraft wake vortex state-of-the-art \& research needs, 2015). In addition, there are some studies on prevention of wake vortex encounters through an airborne wake vortex prevention system (Aircraft wake vortex state-of-the-art \& research needs, 2015; Sölch et al., 2016) and control of aircraft in the event of wake vortex encounter (Schwarz \& Hahn, 2011; Yu, \& Zhang, 2018).

The common practice of industry to identify wake vortex encounters, and other flight events such as wind shear and turbulence, is based on pilot reports. Hence, in many cases this identification is subjective. The reporting procedure involves supplying critical data to a specialized airline analyst. The data include: type of airplane, atmospheric condition, altitude, speed, separations, airplane configuration, flight phase, and other airplane parameters. The analysis is also aided by radar and metrological data. Then, an analyst specialized in flight data recorders (FDR's) conducts a manual examination of the relevant flight data to check if an airplane vortex was encountered. This analysis of pilot reports and FDR data, which are collected for many flights and event encounters over the years, helps to scrutinise the suitability of the imposed vortex-airplane separations (Critchley \& Foot, 1991).

The disadvantages of this procedure are that it involves a subjective human judgment, which may lead to wrong classification of flight events. Some pilots may report mistakenly a vortex encounter due to lack of criteria that can be used to judge with certainty a real vortex encounter. For example, a vortex induced roll angle of less than 5 degrees at an altitude of less than $200 \mathrm{ft}$ may result in an encounter report whereas the same angle at an altitude above $1000 \mathrm{ft}$ will not be noticed. FDR manual analysis agreement with pilot reporting of vortex encounters is in the range of $55 \%-70 \%$ (Woodfield, 1996).

Wake vortex identification was attempted by Woodfield (1998) using pattern searching methods. It was reported that the method has a success rate of about $71 \%$ compared to the manual analysis. However, the method was developed using only 21 examples. Many of the flight research is based on wind tunnel and flight data collected during flights devoted to a particular investigation, which is both risky and expensive. It is also done through virtual flight-testing, theoretical and analytical techniques (Huang, 2015). The speed brake, the airdrop and the landing gear effects have been identified using a nonlinear maximum likelihood parameter estimation method
(Jategaonkar, 1997). The lateral-directional behaviour in stall has also been identified from flight data using the same technique (Singh, 1995). Therefore, it is desirable to investigate simple methods for flight event analysis.

Flight data analysis can be helpful in a number of other tasks: early detection of failure, monitoring performance, and investigating accidents and incidents including vortex encounters. It helps to do a trend investigation to point out important safety concerns that can guide the revision of aviation regulations (Flight Data Services, 2017). Enhancing flight data analysis and pilot reporting of flight events can greatly improve airplane maintenance since it eliminates the performance of needless airplane repairs. For instance, if an un-commanded airplane roll is declared, then maintenance personnel may check and unnecessarily remove parts such as autopilot and yaw control units. This procedure can be avoided if it was reported that the airplane roll was due to a vortex encounter.

An investigation has shown that $47 \%$ of a group of pilots stated that they write fewer reports than required (Haslbeck, Schmidt, \& Schubert, 2015). The reason was the complexity of writing reports with a complicated and time-consuming procedure. Furthermore, based on their reports, pilots receive negative feedback by their superior. There is also reflectance to initiate procedure modifications due to feeling of the insignificance of reporting (Haslbeck et al., 2015). This shows the vitality of pilot reporting training and enhancement of flight event analysis.

This research gives guidance and criteria which can help to carry out a systematic analysis of flight data to reduce subjectivity, decrease analysis time and increase accuracy. The process to establish vortex encounter criteria can also be useful to improve the accuracy of identification of various flight events including: hard landing, wind shear, over controlling, turbulence, etc. The outcome of this research is also useful to other wake vortex and aerodynamic investigations, such as air refuelling, close formation flights and en-route vortex encounters (Woodfield, 1999).

\section{Content of pilot reports}

In this paper 54 pilot reports from a major European airline have been investigated and the accuracy of pilot reports to identify wake vortex encounters is evaluated. The reports cover various flight scenarios and hence many parameters are deduced and analysed which helps enhancing the applicability of the established criteria.

Examination of records has shown that pilot reporting is subjective and in some cases erroneous. It was reported by Woodfield (1996) that a manual analysis of a 100 events reported by pilots as trailing vortex encounters revealed that about $40 \%$ of records were events due to other forms of disturbance. Pilots have the tendency, in particular during final stage of an approach to a busy airport, to blame natural flight events like trailing vortices for the majority of un-commanded disturbances and, sometimes, unexpected commanded disturbances induced by pilots. Stewart (1998) investigated pilot response where 
14 airline pilots flew almost 1000 approaches in the B737100. He stated that pilots thought that the low-level aircraft responses to their own control inputs were responses due to a low-strength vortex disturbances.

\subsection{Description of reported data}

The reports which are provided by pilots after each flight give useful data that could be used to analyse the various flight events. These reports contain: airplane speed, height, type, time, date, configuration, phase, incidence description, airplane control responses, and many other data. In addition, they provide weather data such as visibility, wind direction, wind speed, temperature, etc. Such data is useful to study the influence of weather on the strength and deterioration of vortices and the airplane response to various flight events.

Pilot reports provide information, which is directly relevant to airplane vortex incidence. This information includes encounter severity, change in altitude, variation in roll, pitch, yaw, acceleration, pilot response, control stick shaking, buffet and vibration. Furthermore, the reports give data related to preceding airplane including its type and the separation distance, which supports the analysis by estimating the preceding airplane vortex size, strength and encounter severity. In these reports, pilots are also required to write down their reasons for suspecting a vortex encounter as the cause of the disturbance and they are required to describe the event in words, its cause, results, actions, and preventive measures.

\subsection{Established criteria}

Throughout this research, each record is assigned probability values of $1,0.5$ and 0 to indicate definite, possible or non-vortex encounter respectively. This is accomplished based on a number of criteria, which are established by analysing the data from pilot reports. The classification of records is then compared with pilot reporting of wake vortex encounters, which revealed low accuracy percentages of pilot reporting. Classification criteria are grouped into statistical criteria, atmospheric criteria and derived criteria. The following sections discuss each criteria in detail and present its effect on vortex encounter.

\section{Statistical criteria}

Statistical criteria include leading and following aircraft types, flight phases, encounter altitudes, aircraft configuration and response parameters. The individual statistical criteria are discussed next in terms of its logic, derivation and its application to the available records.

\subsection{Aircraft type}

As shown in Figure 1 below, the investigated 54 records contain 14 types of vortex generating airplanes and 7 types of vortex encountering airplanes. It is clear from Figure 1 that some airplanes are more involved in vortex encounters compared to others as leading and/or following airplanes. This criterion shows that some airplanes are more vulnerable to vortex encounters. In addition, it shows that some airplanes can generate hazardous vortices more than other airplanes.

Furthermore, Figure 1 indicates that B747 and B757 airplanes are involved in 12 and 9 vortex records respectively as vortex-generating airplanes. Concerning the B747 airplane, the finding is logical because it is the heaviest of all the airplanes revealed in Figure 1. In addition, the possibility of airplane vortex encounter relies on the magnitude of the vortex pair, which is around $200 \mathrm{ft}$ deep and $400 \mathrm{ft}$ wide for a B747 (Woodfield, 1999). The B757 airplane has the second highest encounter number as a vortex-generating airplane despite the fact that it is categorised as a medium weight airplane. The A310 is categorized as a heavy airplane but it is involved in one record only as a leading airplane. Critchley and Foot (1991) confirm similar findings. They have investigated 515 vortex encounters where they concluded that the rate, and not the number, of incidents involving B757 and B747 as leading airplanes are higher than other airplanes and even higher than the heavy A310 airplane. As shown in Figure 1, B737 airplane is involved in the largest number of encounters, i.e.16 records in this case, as an encountering airplane. Similar conclusion was reached by Critchley and Foot (1991).

As shown in Figure 1, vortex-generating aircraft are classified into three groups: the first group contains those aircraft with 12 to 7 times being a leader and are assigned a high probability value of 1 . The other two groups have moderate and low probability of involvements. Involvement as a follower aircraft is analysed similarly to classify aircraft into three groups. Accordingly, the individual aircraft in the 54 reports are assigned a value of $1,0.5$, or 0 based on their number of involvements as a generating or encountering aircraft as shown in Table 1.

Table 1. Number of leaders and followers involvement in vortex encounters

\begin{tabular}{|c|c|c|c|}
\hline \multicolumn{2}{|c|}{ Number of involvements } & \multirow{2}{*}{$\begin{array}{c}\text { Encounter } \\
\text { probability }\end{array}$} & Classification \\
\cline { 1 - 2 } Leader & Follower & & \\
\hline 12 to 7 & 16 to 6 & Highly & 1 \\
\hline 6 to 4 & 5 to 3 & Possibly & 0.5 \\
\hline 3 to 0 & 2 to 0 & Unlikely & 0 \\
\hline
\end{tabular}

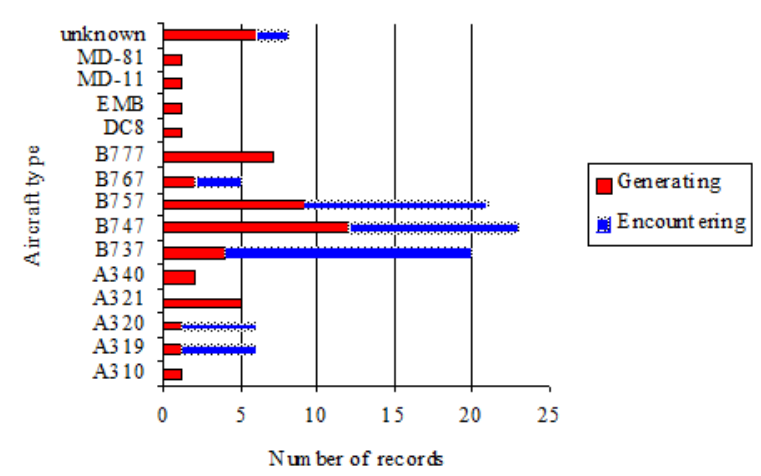

Figure 1. Number of airplane engagements in vortex-encounter records 


\subsection{Flight phases}

Since some flight phases such as take-off, landing and approach are more confined than other phases, then the possibility/probability of vortex encounter is higher during these phases. More airplane-vortex encounters are found in the close airport proximity due to airplane congestion and dense air traffic. Therefore, it is expected that possibility of airplane-vortex encounter is varying depending on flight phases. This matter was examined for all the collected encounter records and three classes were identified.

Figure 2 shows that the number of vortex encounters is highest during approach and climb, where there are 19 and 12 encounters respectively. While descent flight phase has 7 encounters, cruise and landing phases have only two encounters each. Hence, records with encounters occurring at approach and climb are assigned an encounter probability of 1 . Records with incidents at take-off and descent are assigned a value of 0.5 while cruise and landing records are assigned 0 . There are 3 records missing statement of flight phase.

Critchley and Foot (1991) stated that various airplanevortex encounters are occurring during approach phase. This is in agreement with the conclusion from this investigation. As shown in Figure 2, take-off phase has higher vortex encounters than landing despite the fact that they cover the same range of altitude. This case can be justified since airplane vortices usually descent under the flight path of the leading airplane and they seldom move up the flight path (Woodfield, 1999). In addition, vortex encounter at low altitude is affected by rebound in ground proximity (Critchley \& Foot, 1991; Holzäpfel \& Stephan, 2016).

\subsection{Vortex encounter altitude}

The probability of a vortex encounter varies with not only flight phase but also altitude. Encounter altitudes are shown in Figure 3 for a value up to $8000 \mathrm{ft}$ in the main plot area. For clarity, higher altitudes are shown at the right top corner of Figure 3. From Figure 3, the highest density region appears to be in the range of $0-500 \mathrm{ft}$ and another region confined to the $3000 \mathrm{ft}$ line.

This is clarified by counting the number of points in selected altitude ranges. Figure 3 shows that a $500 \mathrm{ft}$ step is reasonable to define the altitude ranges. This value is of the same order of magnitude as the estimated maximum

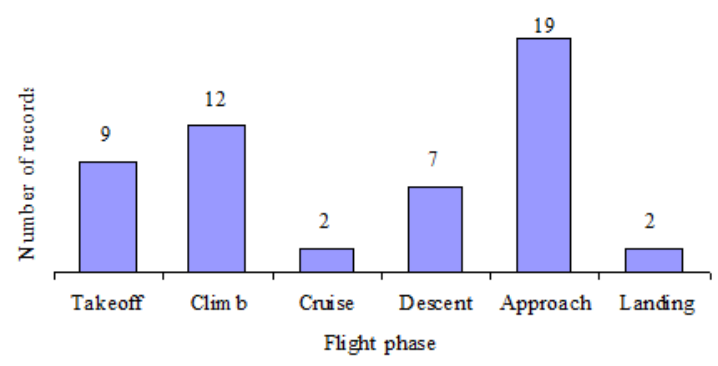

Figure 2. Records encounter classification with respect to flight phases

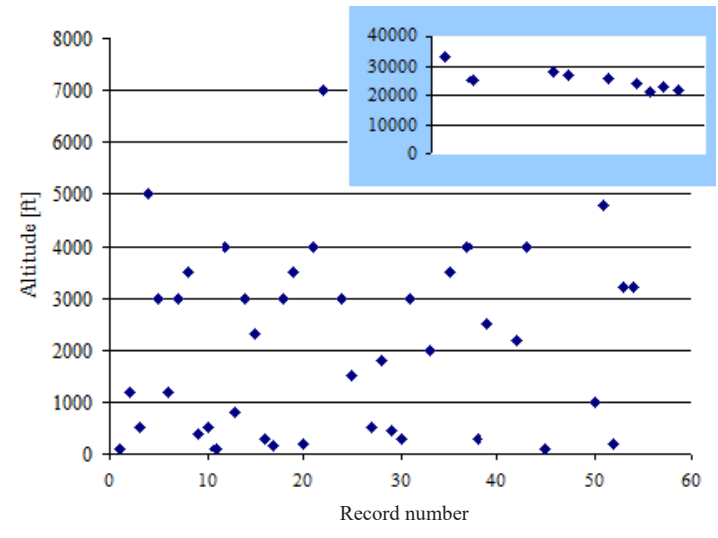

Figure 3. Records distribution based on encounter altitude

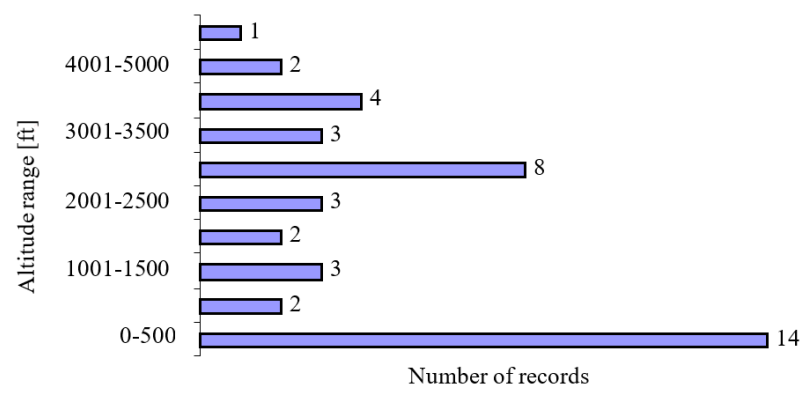

Figure 4. Records classification based on encounter altitude

value of reporting error where it is uncertain if the reported values are radio or pressure altitude.

As shown in Figure 4, there are 41 records that are classified/clustered based on height range and up to 5000 $\mathrm{ft}$. Altitudes which are more than $5000 \mathrm{ft}$ are shown in the auxiliary upper plot of Figure 3.

From Figure 4, it is clear that the $0-500 \mathrm{ft}$ range has the highest density with 14 encounters. The 2501-3000 ft range has 8 records/encounters. These two ranges are assigned probability 1 and the remaining ranges are assigned probability 0.5 . Altitudes ranges greater than $5000 \mathrm{ft}$ are assigned 0 since points are noticed to be scattered over a wide altitude range. This result is logical since there are more flight paths at higher altitudes and hence arbitrary encounters are predicted. Out of all the collected records, 12 records are lacking altitude data.

\subsection{Aircraft configuration}

This criterion is about some parameters including engagement of flaps/spoilers/slats and the use of autopilot. Figure 5 indicates the state of these parameters as listed in the investigated records. There are three parts in each line reflecting if that parameter is engaged/disengaged/ unknown. The Figure indicates that 4 records show that airplanes were turning, 33 were not turning and 17 with unknown status since this information was not reported by all pilots. Based on these numbers, each record is assigned a value of $1,0.5$ and 0 respectively. Figure 5 shows that autopilot was engaged in 21 cases and disengaged in 


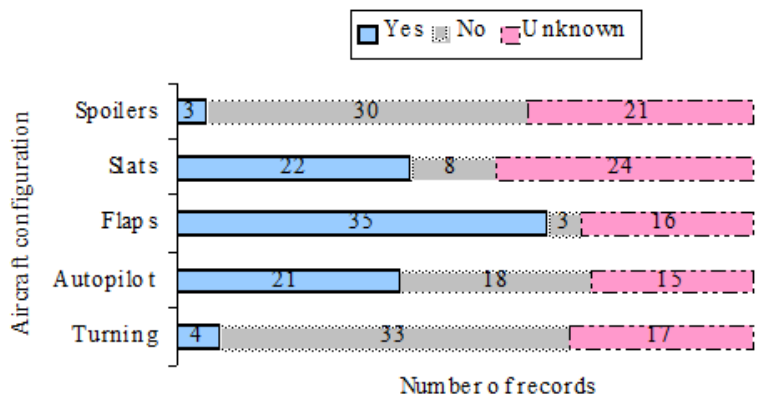

Figure 5. Distribution of records according to aircraft configuration parameter

Table 2. Rounding up the average values

\begin{tabular}{|l|c|}
\hline \multicolumn{1}{|c|}{ Result } & Classification/Probability \\
\hline $0 \leq$ average $<0.4$ & 0 \\
\hline $0.4 \leq$ average $<0.7$ & 0.5 \\
\hline $0.7 \leq$ average $\leq 1$ & 1 \\
\hline
\end{tabular}

other 18 cases. Accordingly, records showing that autopilot engaged or disengaged are assigned a value of 0.5 since records are almost split in half for this parameter. This autopilot criterion assignment of 0.5 is very useful for aggregation of the effect of all criteria when establishing automatic identification techniques such as fuzzy logic, neuro-fuzzy and other artificial intelligence techniques.

Similarly, there are 35 records with flaps on and 3 flaps off and are assigned probability value of 1 and 0.5 respectively and 0 for unknown flaps status. Slats engagement is treated exactly like flaps and spoilers are treated exactly like the turning condition.

The average for all probability values of these five parameters is calculated and then rounded up according to Table 2 to give the collective probability for this criterion for each record.

\subsection{Response parameters}

The investigated pilot reports contain seven parameters relevant to airplane response to a vortex encounter. Figure 6 shows these parameters and the number of times they are stated to vary due to a vortex encounters. It is apparent that roll angle has the highest number of variation. Comparable conclusion was reached by other studies (Stewart, 1998; Sammonds, Stinnett, \& Larsen, 1976). These studies show that airplane roll angle gives the best relationship with the pilot judgement of vortex encounters and its severity. This is even more correct when roll angle is considered in correlation to altitude. The other parameter, which is reported to change more often, is the buffet. Records showing changes in any of these two parameters are assigned encounter probability of 1 . Records showing changes in stick shake or pitch angle were assigned 0.5. Records showing changes in vertical acceleration, altitude change, or yaw were assigned 0 . Each record was assigned the highest values in its response-parameters probabilities.

Vertical acceleration is usually apparent during a perpendicular vortex encounter and sometimes with light roll angle while clear roll angle is apparent during a parallel vortex encounter with sometimes light vertical acceleration. Actual encounters can also be mixed of both vertical and parallel encounters. Therefore, the above analysis is consistent with the classification based on phases since high number of vortex encounters are reported while airplanes are flying within restricted paths. Therefore, it is concluded that the majority of vortex encounters are almost parallel ones.

\section{Atmospheric criteria}

Atmospheric criteria include wind speed and visibility. Atmospheric conditions, in particular wind, affect the structure and lifespan of wake vortices. Furthermore, the separation distances are imposed (regulated) when instrument flight rules (IFR) apply. Under visual flight rules (VFR) pilots are free to choose their own spacing, which should be close to the imposed separations. In reality, however, pilots deviate from these values depending on many factors including wind speed and visibility conditions.

\subsection{Wind speed}

When wind speed increases, the airplane vortices are pushed out of the flight path. This is normally connected with atmospheric turbulence that causes faster vortex decay. Figure 7 shows various wind speeds that are categorized as low, medium and high based on the reported values as listed in the investigated records. There are 27,

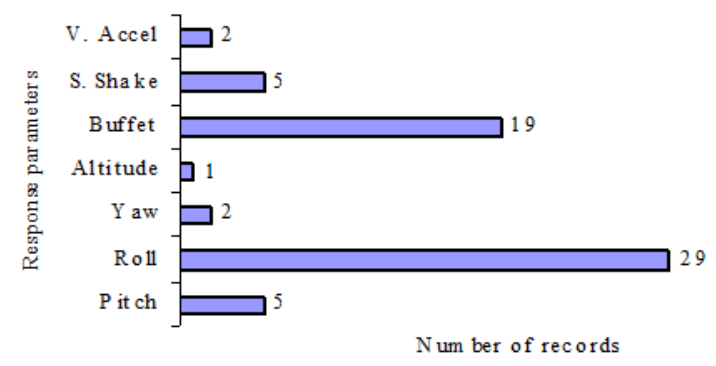

Figure 6. Response parameters versus number of times being changed in encounter records

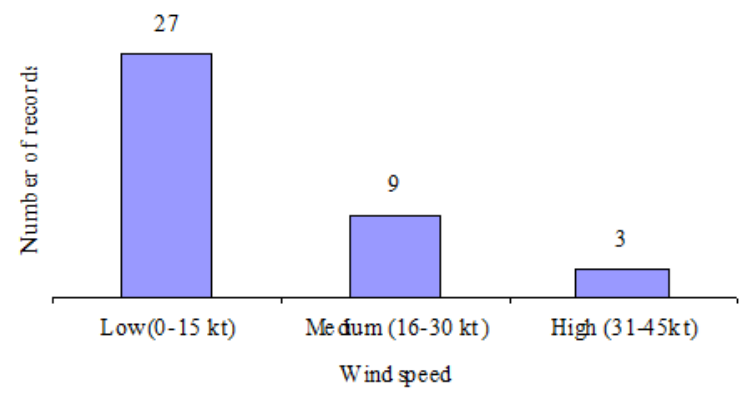

Figure 7. Records distribution according to reported wind speed 
9, and 3 records classified as low, medium and high wind speeds respectively. They are assigned vortex encounter probability/possibility values of $1,0.5$ and 0 respectively. Records with the term "calm" are assigned a value of 1 . There are 15 records which lack wind speeds hence they are given a value of 0 .

\subsection{Visibility}

When VMC (visual meteorological condition) rules are applicable, pilots can choose the suitable separations based on visually spotting the vortex-generating airplane. In such cases, the possibility of vortex encounter event increases as the visibility decreases. Figure 8 indicates three different groups of vortex encounter records in relation to visibility. It is clear and logical that most encounters happened at low visibility with 34 records at VMC. Only 3 vortex encounter records occurred during IMC which are assigned a probability value of 0 since they do not depend on visibility. Moderate visibility accounts for 4 records and were assigned a probability value of 0.5 . Only one record has a high visibility and was assigned probability 0 .

\section{Derived criteria}

Derived criteria are more reliable to validate a vortex encounter because they rely more on numerical data. They include induced roll angle versus altitude, separation distances, and separation times.

\subsection{Induced roll angle versus altitude}

It is found from this study that at low altitudes, airplane vortex encounters are reported by pilots at both small and large roll angles. On the other hand, at high flight altitude, pilots tend to report vortex encounters that only causing high roll angles. Woodfield (1996), has analysed many flight data records (FDRs) and found that at flight altitudes below $100 \mathrm{ft}$, pilots may report vortex encounters at even very small roll angles of $2^{\circ}$. Woodfield also stated that the maximum vortex encounter roll angles can be $5^{\circ}$, $12^{\circ}, 30^{\circ}$ at flying heights of $10 \mathrm{ft}, 100 \mathrm{ft}$ and $3000 \mathrm{ft}$ respectively. Therefore, a roll angle of below $5^{\circ}$ at a height of below $100 \mathrm{ft}$ can be as important to report as $20^{\circ}$ roll angle at a height of more than $1000 \mathrm{ft}$. These issues indicate a correlation between roll angle and height.

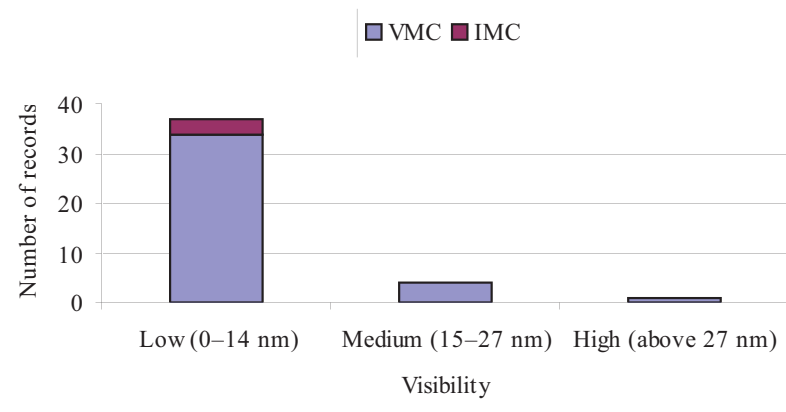

Figure 8. Records distribution based on visibility
Figure 9 below shows roll angles at various altitudes. The main plotting area shows altitudes up to $5000 \mathrm{ft}$ whereas higher altitudes are plotted at the upper right corner of Figure 9. It is evident from the figure that two clusters in the main plot and two in the auxiliary plot can be recognized. The first cluster is for roll angles between $7^{\circ}$ and $15^{\circ}$ at altitude below $1000 \mathrm{ft}$. The second cluster in the main plot is for roll angles between $12^{\circ}$ and $40^{\circ}$ at altitude above $1000 \mathrm{ft}$. Therefore, these two clusters are assigned a probability of 1 . The other two clusters shown in the small plot, one is for low angles below $16^{\circ}$ and the other for high angles between $30^{\circ}$ and $40^{\circ}$. In these two regions, only 5 records are reported over a wide range of altitude between $21000 \mathrm{ft}$ and $33000 \mathrm{ft}$. On the other hand, small roll angles at high altitudes are commonly ignored since they are not hazardous and hence, they are usually not reported. Therefore, small angles at high altitude were assigned a probability 0 while large angles were assigned 0.5 probability.

Zero roll changes are not shown in Figure 9 and are assigned 0 probability since they have not been considered the cause for vortex-encounter reporting.

\subsection{Separation criterion}

The most important criterion of all is based on comparison of actual (reported) separations and imposed (regulated) separations. Imposed separations should be enough to give time for airplane vortices to diminish and be harmless or to be pushed away from flight path. Hence, it is anticipated that the majority of the reported events are at separations below the enforced ones. It is concluded that the lower the actual separations compared to the regulated separations, the higher the probability/possibility that an encounter may occur.

Airplane-vortex separations may be stated in terms of time and/or distance. The normal practice is to use the separation distances as was inferred from the pilot reports. But this practice of using distances can be faulty and confusing because at high speeds the encountering airplane may encounter the vortex before it has diminished to a reasonable level. The final criterion is based on time separations. Airspeed during climb, cruise and descent (high airspeed phases) is higher compared to speeds at other flight phases such as take-off and approach. Hence,

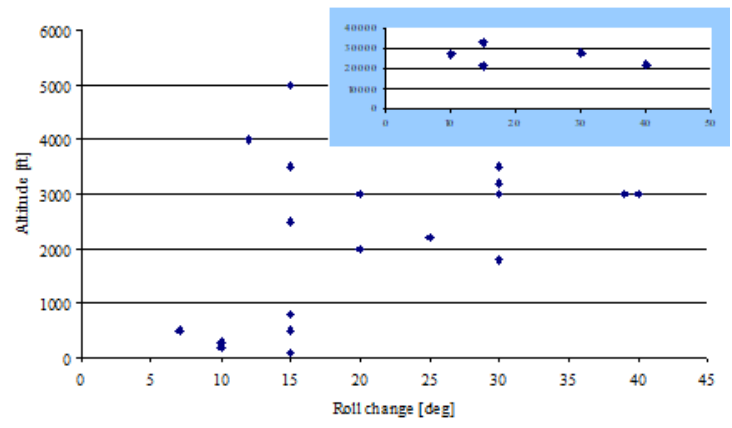

Figure 9. Reported vortex-induced roll angle (absolute) versus altitude 
airplane vortices continue for distances of around 20 nautical miles $(\mathrm{nm})$ during high speed phases and from 5 to $6 \mathrm{~nm}$ at take-off and approach phases while considering calm weather conditions (Woodfield, 1999).

\subsubsection{Separation distance}

The main plot in Figure 10 below reflects the imposed/ regulated separations versus the separations reported by pilots. The plot at the right top corner of Figure 10 reflects that in 26 encounter records pilots did not report specific separation values. There are 18 records that have separations more than the imposed/regulated separations. There are 6 records that show separations equal to the imposed/ regulated separations and only 4 records that have separations below the imposed ones. Hence, most records report separations which are higher than the imposed ones. Therefore, there is no reason to consider these cases as actual vortex encounters and only the reported 4 records are possible candidates for vortex encounters. The separation distance criterion can be ignored in this case; and the time separations can be considered as the influential criterion because it indicates whether the airplane vortices have sufficient time to diminish and/or move away from the flight track.

\subsubsection{Time separation}

Speeds and separation distances are reported and therefore, the time separations can be calculated where time = distance/speed. The speed used for calculations is the true speed, which does not include the effect of wind speed. As a result, the calculated separation time has inaccuracy; since, for example, the presence of head wind will result in low separation. Once wind speeds and directions are available for all investigated records, the calculated separations can be updated which will be done in future investigation. Figure 11 shows that time separations indicate the real case of the investigated encounter records. As shown in the figure there are 25 records with time separations below the imposed time separations. Figure 11 (small plot) indicates that 3 records have time separations more than the imposed/regulated time separations in comparison to a total of 18 records depending on distance separations as previously discussed. To make classification more ac-

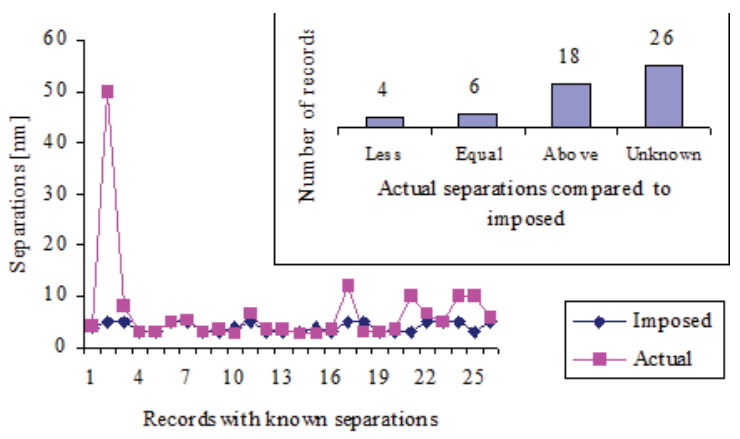

Figure 10. Reported separations compared to the UK imposed separations

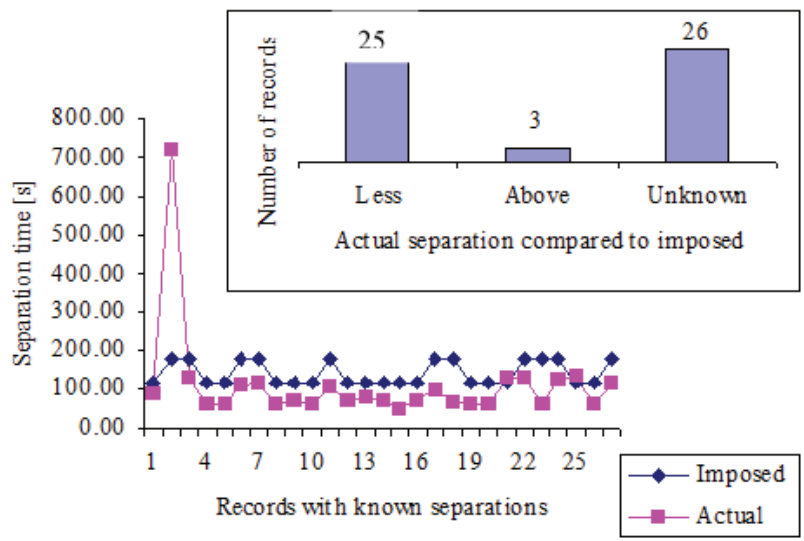

Figure 11. Reported time separations compared to the UK imposed separations

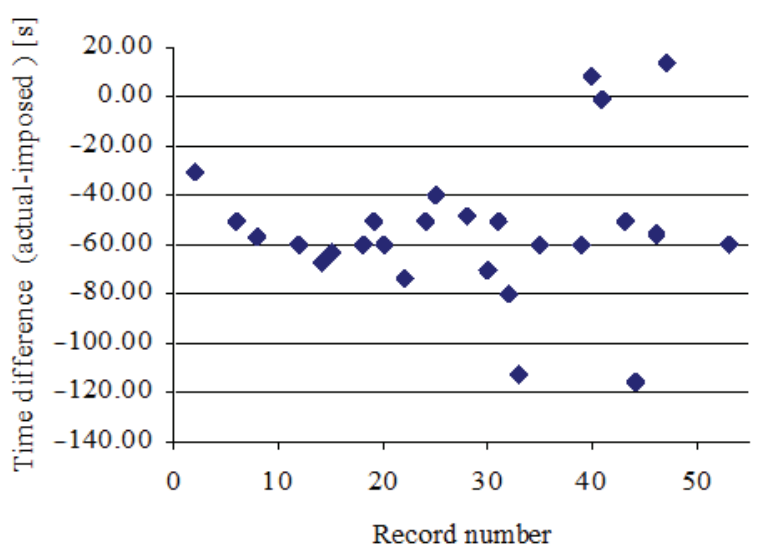

Figure 12. Difference between imposed and actual time separations

curate, the difference between imposed and actual time separations was calculated as shown in Figure 12.

Records with time difference less than $-30 \mathrm{~s}$ (all points below 0 line in Figure 12) are assigned probability 1. Three records are located above but close to the zero difference line are assigned 0.5 probability. One record with an actual time separation of $720 \mathrm{~s}$ compared to imposed separation of 180 s, not shown in Figure 12 for clarity, is assigned probability 0 the same as records with unknown separations. It is to be clear that the imposed time separations is not calculated but it is set by regulations (Hinton, 1997; Critchley \& Foot, 1991). UK minimum imposed time separation is 3 minutes between a heavy leading and a medium following aircraft (Wake turbulence, 2015).

\section{Results and discussions}

Al-Mahadin and Bouslama $(2017,2018)$ have constructed artificial intelligence (AI) vortex identification models by utilizing data from flight data recorders for 210 flight records including the ones corresponding to the investigated 54 pilot reports. An average success rate of identification of $83.7 \% 84.2 \%$ were obtained from the two investigations respectively. These AI models were also utilized to validate 
the 54 pilot reports which helped to evaluate the pilot reporting accuracy and also to support the uniformity of the established criteria.

\subsection{Analysis of pilot reports}

Analysis of the available pilot reports revealed 10 criteria, which are used to classify the records, as shown in Table 3. As an example, one of the criteria selected for record classification is based on flight phase, which revealed that approach, followed by climb, had the highest numbers of records identified as vortex encounters. This is consistent with the fact that wake vortex encounters are more likely to be reported at the vicinity of airports because of dense air traffic, which is constrained into corridors, and because of increased pilot alertness. Another highly reliable criterion was the visual recognition of leading airplanes near the flight tracks and at a distance not far enough to allow vortex dissipation. This criterion was implemented through the visibility and separation criteria. Most records, which satisfied these two criteria, had a collective classification of 1 , as shown in Table 3.

In Table 3 each contributing criterion is assessed individually. For example, if the lead aircraft is a heavy aircraft, such as a Boeing 747, then value 1 is entered for the particular record in the first row of the Table. All factors are then combined by calculating the average of assessments for all criteria and then rounding the value according to Table 2 to give the overall classification as shown in the last two rows of Table 3. Classification 1 corresponds to equal weightings for all criteria while classification 2 has double weight for the time separation criterion. It is anticipated that time separations criterion is more influential and reliable than other criteria since it is the determining factor to allow time for vortex dissipation. Classification 2 is obtained by multiplying the probabilities of time separations by 2 and added to values for other criteria and then divided by 10 . This process of criteria weight adjustment can be implemented if new information is provided to indicate that some criteria are more influential than others. Furthermore, it can work the other way through multiplying by a value of less than 1 if a criterion is proven less influential.

Table 3 shows only a sample of 9 records while all 54 records are classified similarly and results are shown in Figure 13 below.

\subsection{Typical records}

Record 1 shown in Table 3 is of interest because the pilot's comments indicated a conflict with the air traffic controller (ATC) as stated in the pilot report. While the pilot has reported moderate wake turbulence from preceding A321 aircraft during landing, the ATC controller did not believe wake turbulence from the A321 in the presence of a tail wind could be a factor. The generating aircraft type is used as a classification criterion where the A321 aircraft is rated at a probability value of 0.5 since it is reported less times as a generator compared to other aircraft such as B747. Wind speed is also used as a classification criterion and for this record, it is assigned a value of 1 , since the wind speed is reported to be only 7 knots which is considered a low wind speed compared to other reported values. It should be noted that during low wind speed conditions the trailing vortices are more persistent whereas high wind speed causes faster vortex breakdown and therefore, encounter probability is expected to be high in the first case and low in the second. The result for this record is a value of 0.5 , indicating a moderate possibility of vortex encounter if equal weight is given to all criteria. If double weight is given to the time separation criterion (classification 2 in Table 3 ) then it is also given a probability value of 0.5 . Therefore, the derived criteria agree with the pilot report summary, which included some uncertainty comments.

Another interesting record is number 4 in Table 3 for which a time separation of 12 minutes is reported,

Table 3. Records classification based on criteria derived from pilot reports

\begin{tabular}{|c|l|c|c|c|c|c|c|c|c|c|}
\hline \multirow{2}{*}{$\#$} & \multirow{2}{*}{ Criteria } & \multicolumn{9}{c|}{ Number of records } \\
\cline { 2 - 12 } & & 1 & 2 & 3 & 4 & 5 & 6 & 7 & 8 & 9 \\
\hline 1 & Generating aircraft & 0.5 & 1 & 1 & 0 & 0.5 & 1 & 0 & 1 & 1 \\
\hline 2 & Encountering aircraft & 0.5 & 1 & 1 & 0.5 & 0.5 & 0.5 & 1 & 1 & 1 \\
\hline 3 & Flight phase & 0 & 1 & 1 & 1 & 1 & 1 & 1 & 1 & 0 \\
\hline 4 & Altitude & 1 & 0.5 & 1 & 0.5 & 1 & 0.5 & 1 & 0.5 & 0 \\
\hline 5 & Response parameters & 0 & 1 & 0.5 & 1 & 1 & 0 & 1 & 1 & 1 \\
\hline 6 & Roll \& altitude & 0 & 0 & 0 & 1 & 1 & 0 & 1 & 1 & 0 \\
\hline 7 & Configuration & 0.5 & 0.5 & 1 & 0 & 0.5 & 1 & 1 & 1 & 0 \\
\hline 8 & Wind speed & 1 & 1 & 1 & 0 & 0 & 1 & 1 & 0.5 & 0 \\
\hline 9 & Visibility & 1 & 1 & 1 & 0 & 1 & 1 & 1 & 1 & 0.5 \\
\hline 10 & Time separations & 0 & 1 & 0 & 0 & 0 & 1 & 0 & 1 & 1 \\
\hline & Classification 1 & 0.5 & 1 & 1 & 0.5 & 0.5 & 1 & 1 & 1 & 0.5 \\
\hline & Classification 2 & 0.5 & 1 & 0.5 & 0 & 0.5 & 1 & 1 & 1 & 0.5 \\
\hline
\end{tabular}


compared to the imposed separation of only 3 minutes. Therefore, it is expected that this record should have a low encounter probability. Considering all ten criteria, a probability value of 0.5 is obtained when time separation is given the same weight as other criteria. However, it is believed that the time separation criterion is the most reliable of all criteria and should be assigned a higher weight. Therefore, double weight is assigned for the time separation criteria and results are shown in Table 3 as classification 2, which shows a value of 0 for this record.

Record number 9 is a reported encounter at $12 \mathrm{~nm}$ separation. Initial analysis indicates a non-vortex encounter due to long separation distance. However, the important separation criterion is the time and not the distance. It is found that, at this separation and a Mach number of $0.74(906.5 \mathrm{~km})$, the time separation is about 100 seconds compared to an imposed time separation of 180 seconds. According to this criterion, therefore, it is given a probability of 1 , although the collective probability, according to all criteria, is found to be only 0.5 . One of the reasons to degrade this record encounter probability is the existence of a high wind speed of 43 knots.

\subsection{Overall results}

The collective probability for each record is calculated by taking the average of probabilities of all criteria and then rounding the value according to Table 2 . Overall results are shown in figure 13 where records are distributed among the three classes 1, 0.5 and 0 with 23, 24 and 7 records respectively. When giving double weight (classification 2), records were redistributed as 21, 25 and 8 records respectively.

While pilot reporting has indicated that 54 flights events are vortex encounters, this research has shown that only 21 are confirmed vortex encounters. Therefore, pilot reporting accuracy is about $40 \%$. If half of the " 0.5 classification" records are considered vortex encounters then total encounters will be 33 , which makes reporting accuracy about $62 \%$, which is still low. This finding agrees with other investigations where it is stated that manual analysis agreement with pilot reporting is in the range of $55 \%-70 \%$ (Woodfield, 1996, 1998) due to pilot reporting deficiency.

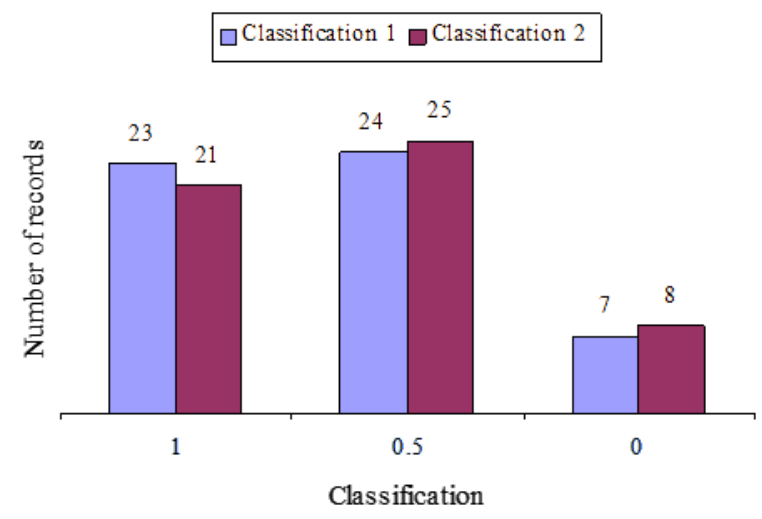

Figure 13. Records classification according to all criteria

\section{Conclusions}

Major airlines require their pilots to report flight events such as wind shear, hard landing and airplane vortex encounters. FDR analyst investigate the relevant FDR data to confirm/negate the reported flight events. This process of reporting and analysis is subjective, complicated and consumes effort and time. Investigations reveal that there is a considerable difference between pilot flight-event reporting and FDR data analysis. This disagreement can adversely affect flight safety and airline effectiveness. This paper shows that accuracy of pilot reporting is in the range of $40-62 \%$, which requires measures to ensure the required level of safety and efficiency.

In this research, a number of criteria were established to support the systematic analysis of flight data to identify flight events with higher accuracy. Results from this investigation and other research (Al-Mahadin, \& Bouslama, 2017, 2018, 2019a, 2019b) can aid the adjustment of airplane vortex separations which can reduce hazardous vortex encounters and enhance the capacity of various busy airports. Recognition of the investigated criteria by airline pilots will improve their reporting skills and increase the identification accuracy of the various flight events.

Accurate, systematic and reliable analysis of flight data and hence flight events identification could save hundreds of hours per month at one airline where many airplanevortex encounters are flagged. The data used in this research cover a range of flight conditions; hence, the criteria can be applied for other aircraft and conditions.

The developed criteria may aid the establishment of automatic identification of various flight events utilizing artificial intelligence techniques (Al-Mahadin, \& Bouslama, 2017, 2018, 2019a, 2019b). The identification of vortex encounters from this investigation can be utilized to study the recognition of other flight events including hard landing, wind shear, turbulence, etc.

This investigation has revealed vital results that are recommended as follows:

1. Using the developed criteria to aid analysis of flight data which can help flight-events identification.

2. Introducing procedures to improve the accuracy of pilot reporting of flight events through awareness and utilization of the established criteria.

3. Using the established criteria to help the automatic identification of flight events using computer techniques such as artificial intelligence.

4. Revising vortex separations based on results from various relevant research including the established criteria and other manual and automatic techniques, which may improve safety and airport capacity.

5. Investigating the presented technique to enhance identification of other flight events.

\section{Acknowledgements}

Many thanks to Higher Colleges of Technology HCT/UAE for supporting this research. 


\section{References}

Aircraft wake vortex state-of-the-art \& research needs. (2015). WakeNet3-Europe EC Grant Agreement No. ACS7GA-2008-213462.

Al-Mahadin, A., \& Bouslama, F. (2017). Automatic identification of wake vortex traverse by transport aircraft using fuzzy logic. Proceedings of the IEEE 4th ISCMI (pp. 133-139). Port Louis, Mauritius. https://doi.org/10.1109/ISCMI.2017.8279613

Al-Mahadin, A., \& Bouslama, F. (2018). Neuro-Fuzzy techniques for the identification of aircraft wake vortex encounters. Proceedings of ASET 2018. Dubai, UAE. https://doi.org/10.1109/ICASET.2018.8376808

Al-Mahadin, A., \& Bouslama, F. (2019a). Airplane vortex encounters identification using multilayer feed-forward neural networks. International Journal of Machine Learning and Computing (IJMLC), 9(1), 1-7. https://doi.org/10.18178/ijmlc.2019.9.1.1-7

Al-Mahadin, A., \& Bouslama, F. (2019b). Recognition of airplane wing-tip vortices encounters using neural networks. International Journal of Machine Learning and Computing (IJMLC), 9(2), 115-120. https://doi.org/10.18178/ijmlc.2019.9.2.774

Chernyshev, S., Gaifullin, A., \& Sviridenko, Y. (2014). Civil aircraft vortex wake. Progress in Aerospace Sciences, 71, 150-166. https://doi.org/10.1016/j.paerosci.2014.06.004

Critchley, J., \& Foot, P. (1991). United Kingdom civil aviation authority wake vortex database: analysis of incidents reported between 1982 and 1990. London, UK.

Current market outlook 2012-2031. (2012). The Boeing Company, Seattle, USA.

Flight Data Services. (2017). FDM/FOQA services: a proactive approach to safety. https://www.flightdataservices.com/fdmfoqa-services/flight-data analysis

Haslbeck, C., Schmidt, M., \& Schubert, E. (2015). Pilots' willingness to report aviation incidents. Researchgate, conference paper.

Hinton, D. (1997). Aircraft vortex spacing system (AVOSS) concept and development; CP-97-206235 (ed.), Proceedings of the NASA first wake vortex dynamic spacing workshop. Langley Research Centre, Hamton, VA, USA.

Höhne, G., Fuhrmann, M., \& Luckner, R. (2004). Critical wake vortex encounter scenarios. Airbus Deutschland $\mathrm{GmbH}$, Kreetslag 10, 21129, Hamburg, Germany. https://doi.org/10.1016/j.ast.2004.07.005

Holzäpfel, F., \& Stephan, A. (2016). Wind impact on single vortices and counterrotating vortex pairs in ground proximity. Flow, Turbulence and Combustion, 97, 829-848. https://doi.org/10.1007/s10494-016-9729-2

Huang, M. (2015). A review of wind tunnel based virtual flight testing techniques for evaluation of flight control systems. In- ternational Journal of Aerospace Engineering, 2015(1), 1-22. https://doi.org/10.1155/2015/672423

Huang, M., Zhang, Z., \& Cui G. (2017). Numerical study of aircraft wake vortex evolution near ground in stable atmospheric boundary layer. Chinese Journal of Aeronautics, 30(6), 1866-1876. https://doi.org/10.1016/j.cja.2017.08.012

Global market forecast 2012-2031. (2012). Airbus S.A.S., Cedex, France.

Jategaonkar, R. (1997). Identification of speed brake, air-drop and landing gear effects from flight data. Journal of Aircraft, 34(2), 174-180. https://doi.org/10.2514/2.2169

Safety and flight operations: loss of control in-flight accident analysis. (2015). International Air Transport Association (IATA), Montreal, Canada.

Sammonds, R., Stinnett, G., \& Larsen, W. (1976). Wake vortex encounter hazard criteria for two aircraft classes. NASA TM $X-73,113, F A A-R D-75-206$.

Schwarz, C., \& Hahn, K. (2011). Automated pilot assistance for wake vortex encounters. Aerospace Science and Technology, 15, 416-421. https://doi.org/10.1016/j.ast.2010.09.008

Singh, J. (1995). Identification of lateral-directional behaviour in stall from flight data. Journal of Aircraft, 33(3), 627-630. https://doi.org/10.2514/3.46993

Sölch, I., Holzäpfel, F.delmoula, F., \& Vechtel, D. (2016). Performance of on-board wake vortex prediction systems employing various meteorological data sources. Journal of Aircraft, 53, 1505-1516. https://doi.org/10.2514/1.C033732

Stewart, E. (1998). A piloted simulation study of wake turbulence on final approach. AIAA Atmospheric Flight Mechanics Conference and Exhibit. Boston, Massachusetts, USA, AIAA 98-4339. https://doi.org/10.2514/6.1998-4339

Vortex avoidance procedures. (2017). Official guide to basic flight information and ATC Procedures, Aeronautical Information Manual (AIM).

Wake turbulence (2015). Civil Aviation Authority, NATS services. Aeronautical Information Circular p001/2015, United Kingdom.

Woodfield, A. (1996). Analysis of flight data records of reported wake vortex encounters. Woodfield aviation research.

Woodfield, A. (1998). Automatic identification of wake vortex encounters from flight data records. Woodfield aviation research.

Woodfield, A. (1999). En-route encounters with wake vortices, and the implications of reduced vertical separation minima (RVSM). Woodfield Aviation Research, report no. 9901.

Yu, Y., \& Zhang, Y. (2018). Safe control of trailing UAV in close formation flight against actuator fault and wake vortex effect. Aerospace Science and Technology, 77, 189-205. https://doi.org/10.1016/j.ast.2018.01.028 